\title{
Gestión administrativa en el proceso presupuestal del sector educación en gobiernos subnacionales del Perú
}

\author{
Rober Aníbal Luciano Alipio \\ rluciano@unaat.edu.pe \\ Universidad Nacional Autónoma Altoandina de Tarma \\ Tarma- Perú. \\ José Abdón Sotomayor Chahuaylla \\ jsotomayor@unamba.edu.pe \\ David Barrial Acosta \\ dbarrial@unamba.edu.pe \\ Universidad Nación al Micaela Bastidas de Apurímac \\ Abancay-Perú
}

\section{Mercedes Fidelia Loayza Chacara} mloayza@uandina.edu.pe

\section{RESUMEN}

Universidad Andina del Cusco

La presente investigación tiene como objetivo principal determinar la influencia de la gestión administrativa en el proceso presupuestal en una dependencia de un gobierno regional en el Perú; La metodología seguida fue de enfoque cuantitativo, para ello se ha recurrido a un diseño no experimental, de recojo de datos en un solo momento, tomando como informantes a 147 servidores públicos, se aplicó un instrumento tipo Likert a través de la encuesta, se procesó y analizó los datos con estadística descriptiva, utilizando tablas de frecuencias; en el caso de resultados inferenciales se ha determinado a través de la prueba estadística regresión logística ordinal; Los resultados obtenidos muestran que el $61.9 \%$ de encuestados considera que la gestión administrativa es buena y el $51 \%$ considera que el proceso presupuestal es aceptable, así mismo se ha encontrado un seudo R-cuadrado de 0.918 calculado con la prueba de Nagelkerke, con una significancia de 0.000 menor a $\mathrm{p}=0.05$, lo que nos indica que la gestión administrativa explica el $91.8 \%$ del comportamiento de la variable proceso presupuestal; Concluyendo que existe una alta influencia de la gestión administrativa en el proceso presupuestal en una dependencia del sector educación en un gobierno regional en el Perú.

Palabras clave: gestión administrativa; proceso presupuestal; gobierno regional. 


\title{
Administrative management in the budget process of the education sector in subnational governments of Peru
}

\begin{abstract}
The main objective of this research is to determine the influence of administrative management in the budgeting process in a dependency of a regional government in Peru; The one followed was a quantitative approach, for this a non-experimental design has been used, data collection in a single moment, taking 147 public servants as informants, a Likert-type instrument was applied through the survey, it was processed and analyzed the data with descriptive statistics, using frequency tables; In the case of inferential results, it has been determined through the ordinal logistic regression statistical test; The results obtained show that $61.9 \%$ of respondents consider that administrative management is good and $51 \%$ consider that the budget process is acceptable, likewise a pseudo R-square of 0.918 has been found calculated with the Nagelkerke test, with a significance of 0.000 less than $p=0.05$, which indicates that administrative management explains $91.8 \%$ of the behavior of the budget process variable; Concluding that there is a high influence of administrative management in the budget process in a dependency of the education sector in a regional government in Peru.
\end{abstract}

Keywords: administrative management; budget process; regional government.

Artículo recibido: 05 octubre. 2021 Aceptado para publicación: 02 noviembre 2021

Correspondencia: rluciano@unaat.edu.pe Conflictos de Interés: Ninguna que declarar 


\section{INTRODUCCIÓN}

La educación es un derecho fundamental que debe ser garantizado por el Estado, para ello se destinan recursos económicos que deben ser gestionados de forma adecuada, a fin de atender todas las necesidades del sector, siguiendo un principio de prioridad (Ministerio de Economía y Finanzas, 2021). El proceso presupuestal pasa por diversos pasos que son programación, formulación, aprobación, ejecución y evaluación (Ríos et al., 2017); cada paso es importante para lograr los objetivos que se plantea a nivel nacional como a nivel institucional, en los gobiernos regionales se sigue el proceso a nivel de unidades ejecutoras y se consolida a nivel de pliego presupuestal.

En los procesos presupuestales se presenta una serie de dificultades que van desde la participación de los actores, la inadecuada identificación de las verdaderas necesidades por atender, la escasa información real con la que se cuenta acerca de las brechas sin cubrir, así como actos de corrupción en la ejecución, además del escaso compromiso de los responsables en involucrase adecuadamente (Abdulhakeem, 2020).

Hacia el año 2020, según el portal de consulta amigable del Ministerio de Economía y Finanzas, el presupuesto institucional modificado se ha gestionado 15,040,989,825 de soles a nivel de los gobiernos regionales de todo el Perú, de los cuales se ha devengado $14,628,848,279$ de soles, logrando una ejecución del $97.3 \%$, valor considerado como alto (DGPP, 2018).

De acuerdo a la norma los procesos presupuestales deben ser participativos, es decir deben considerar a los ciudadanos como principales actores, los que deben dar su punto de vista y a los que se les debe consultar sobre las principales necesidades por atender así como cuales de esas necesidades se deben priorizar, sin embargo en los últimos años si bien es cierto se realiza actividades de cumplimiento en el papel, más no se involucra adecuadamente a estos actores (Pineda y Pires, 2017). Entre otras las causas que no permiten el involucramiento son la capacitación a los actores, el no cumplimiento de los acuerdos asumidos en los procesos, los retrasos y demoras en la atención de las solicitudes (Pagani y Pantaleon, 2018).

En los últimos años en el Perú se viene aplicando el presupuestos por resultados, principalmente en los sectores de salud y educación, esta metodología se centra en la relación insumo producto, definiendo la producción del sector público en las políticas que se va a implementar, propiciando la aplicación de indicadores verificables en los logros a 
nivel de impactos o resultados obtenidos, buscando las relaciones entre los resultados que se obtiene en función a calidad y cantidad de bienes y servicios que las entidades brindan al ciudadano (Makón, 2007).

Para poder gestionar adecuadamente los recursos públicos que cuentan las instituciones, es fundamental la gestión administrativa, que permita a través de un proceso de planeación, organización, dirección y control de los diversos recursos ya sean físicos, financieros, tecnológicos y humanos, lograr cumplir con la misión y los objetivos institucionales (Voloshyna, 2021).

La teoría que sustenta tanto la gestión administrativa y el proceso presupuestal es la nueva gestión pública, que considera un papel preponderante hacia la tercerización al sector privado de los servicios públicos, alta especialización de funcionarios públicos y un fortalecimiento del Estado en su rol contralor, de tal manera que pueda equiparar fuerzas frente al libre actuar del sector privado, poniendo para ello una normativa que le permita garantizar la libre competencia así como que se cumpla con una prestación eficaz y eficiente de los diversos bienes y servicios hacia la comunidad (Massey, 2019).

Diversos autores han realizado aproximaciones teóricas con la finalidad de conceptualizar la nueva gestión pública, entre ellos se tiene a Hood (1991), quien platea siete elementos doctrinales que se encuentran superpuestos en la gestión pública, la cual debe ser profesional, debe contemplar estándares y medidas de desempeño, poner mayor control en lo que produce, se debe propiciar la desagregación de unidades, buscar una mayor competencia, tratar de incluir practicas del sector privado en la gestión pública y tratar de tener una mayor disciplina y parsimonia cuando se usa los recursos con los que cuenta el sector público. Por tanto, se entiende a la gestión publica como la obtención de diversos poderes a través de los cuales, de manera gerencial se puede controlar y organizar las diversas actividades, con la finalidad que coincidan con las metas y objetivos que se propone la institución (Funck \& Karlsson, 2020).

Por otro lado un gran número de investigadores han tratado de estudiar la influencia de la gestiona administrativa en el tema presupuestal a nivel del sector público, se puede citar a Cotrina (2017) que a través de una investigación correlacional causal, de diseño no experimental, con recolección de datos en un solo momento, aplicando una encuesta a 105 trabajadores administrativos del sector educación a nivel de gobierno nacional, obtuvo que el $51.4 \%$ de los encuestados consideran que la gestión administrativa es 
adecuada y un $47.6 \%$ perciben que la implementación del presupuesto es útil, así mismo se concluye que existe una influencia del $54 \%$ de la gestión administrativa en la ejecución del presupuesto.

Similar estudio fue realizado por Ayala (2018) que se propuso medir la relación entre la gestión administrativa y el presupuesto de un gobierno local a nivel municipal, enfocándolo en lo cuantitativo, con un nivel correlacional, el diseño que aplico en el estudio fue el no experimental, recolectando los datos en un solo momento, la muestra estuvo conformada por 140 trabajadores municipales a los que se les aplico un cuestionario de 23 preguntas a través de la encuesta, concluyendo que existe una relación del 0.729 entre las variables gestión administrativa y presupuesto.

Otro estudio es el que llevo a cabo Del Pozo (2017) que buscó determinar la relación entre la gestión administrativa y el presupuesto por resultados, con un método hipotético deductivo, de diseño no experimental, aplicando una encueta y un cuestionario para obtener los datos de la investigación, concluyendo que existe evidencia empírica para afirmar que la relación entre gestión administrativa y presupuesto por resultados, es positiva.

De igual manera un estudio realizado por Stack (2018), reveló que la capacidad administrativa es necesaria para lograr el éxito de las políticas y prevenir sus fracasos. Sin embargo, una literatura fragmentada, escasa empírica y un enfoque en los países desarrollados han llevado a una brecha en el examen de cómo se debe medir y construir esa capacidad; particularmente en contextos en desarrollo. Los hallazgos sugieren que tanto los académicos como los profesionales deben ser más conscientes del hecho de que el desarrollo de capacidades es un proceso continuo, que requiere una evaluación y medición específicas de los componentes de la dimensión organizacional-operativa. Dichos componentes incluyen la mejora de la autonomía y la competencia administrativas, y arreglos que incentiven la coordinación y la colaboración, al tiempo que salvaguardan el control y la supervisión adecuados.

La investigación plantea la siguiente pregunta general ¿Cuál es la influencia de la gestión administrativa en el proceso presupuestal en un sector del gobierno regional?; del mismo modo se propone determinar la influencia de la gestión administrativa en el proceso presupuestal, en el sector educación en un gobierno regional del Perú; la hipótesis general 
planteada fue que la gestión administrativa influye positivamente en el proceso presupuestal del sector educación en un gobierno regional.

El estudio se justifica debido a que aborda un tema central como es la gestión administrativa de los recursos financieros del sector público; así mismo permitirá arrojar mayores luces teóricas y empíricas para explicar dicha influencia, así mismo permitirá a investigadores tener mayores argumentos científicos para poder explicar este fenómeno; por otro lado se espera contribuir con los administradores públicos en los distintos niveles jerárquicos, a implementar acciones concretas que permitan gestionar de manera más eficiente los recursos escasos, desde su programación, hasta la evaluación final, a partir de una eficiente gestión administrativa de la cosa pública, principalmente en un sector tan importante como es el educativo.

\section{ESTRATEGIAS METODOLÓGICAS O MATERIALES Y MÉTODOS}

El proceso metodológico seguido en la investigación fue de un tipo de investigación básica, puesto que se buscó contribuir en incrementar el conocimiento que se tiene sobre el objeto de estudio (Esteban, 2021); el enfoque en el cual se ha conducido es el cuantitativo, debido a que se ha seguido un procedimiento bien definido y estructurado que ha permitido probar las hipótesis plateadas (Hernández y Mendoza, 2018); el alcance es de una investigación descriptiva-explicativa-causal, ya que se ha descrito tanto las variables como las dimensiones en estudio, del mismo modo se ha explicado la influencia de la variable independiente en el comportamiento de la variable dependiente (Hernández et al., 2014); se ha encauzado en un diseño no experimental, puesto que no fue el propósito manipular las variables de estudio, más bien fue el medir en su estado natural en la realidad, recogiendo los datos en un solo momento (Kesmodel, 2018).

La población estuvo constituida por trabajadores administrativos del sector educación a nivel de gobierno regional de Apurímac, con un total de 235 servidores, de los cuales se extrajo una muestra estadística de 147 colaboradores, aplicando una encuesta virtual; la técnica que se ha utilizado es la encuesta, como instrumento se recurrió a un cuestionario de 56 ítems con escala tipo Likert (Taherdoost, 2020), para la validez del instrumento se ha recurrido al juicio de expertos obteniendo valoraciones de excelente y recomendaciones de aplicabilidad del instrumento, la confiabilidad fue medida utilizando la prueba de Alfa de Cronbach, obteniendo como resultado para la variable gestión administrativa $0.907 \mathrm{y}$ en la variable proceso presupuestario 0.935 , lo que indica alta 
convergencia de los diversos ítems con la variables en estudio (Tuapanta et al., 2019); para el procesamiento y presentación de resultados se ha utilizado tanto la estadística descriptiva a través de tablas de frecuencias, y en lo que concierne a los resultados inferenciales se ha recurrido a la regresión logística ordinal (Navarro et al., 2014); En el proceso de investigación se ha practicado en todos sus extremos los aspectos éticos de participantes informados, equidad de género, respeto de autoría en información secundaria y primaria.

\section{RESULTADOS Y DISCUSIÓN}

A nivel descriptivo los resultados encontrados fueron que un $61.9 \%$ de los encuestados, consideran que la gestión administrativa es buena, seguido por un $25.2 \%$ que consideran que es aceptable y un $12.9 \%$ que manifiesta que es excelente, es decir que existe una percepción en general de favorable hacia esta variable.

Si se analiza las dimensiones se puede evidenciar que el $55.8 \%$ de los encuestados consideran que la planificación es buena, un $27.9 \%$ consideran que es excelente y un $16.3 \%$ consideran que es aceptable, por tanto, se puede afirmar que la planeación es una de las fortalezas de la gestión administrativa en la institución analizada; así mismo un $37.4 \%$ considera que la organización es buena, un 31.3\% considera que esta es aceptable, un $26.5 \%$ considera que es excelente y un $4.8 \%$ consideran que es deficiente, si bien se evidencia cierta inclinación favorable hacia la organización, sin embargo esta está más distribuida hacia el promedio.

En lo que respecta al proceso de dirección es considerado por un $49 \%$ de los encuestados como bueno, un $30.6 \%$ considera que es aceptable, un $16.3 \%$ consideran que es deficiente y el $4.1 \%$ considera que es excelente, en general se puede afirmar que la dirección en la entidad estudiada es considerada en nivel medio, si bien es cierto se acepta como regular queda margen para seguir mejorando; en lo que respecta al control, este es considerado como aceptable por un $46.3 \%$, como bueno por un $40.1 \%$ y como deficiente un $13.6 \%$, por lo que se puede evidenciar que el control es considerado en general como regular, lo que indicaría que se puede seguir mejorando los procesos de control en la entidad estudiada. Estos datos se muestran en la tabla 1. 
Tabla 1

Resultados descriptivos de la variable gestión administrativa y sus dimensiones

\begin{tabular}{|c|c|c|c|c|}
\hline & & Nivel & f & $\%$ \\
\hline \multirow{3}{*}{ Variable } & \multirow{3}{*}{$\begin{array}{l}\text { VI: Gestión } \\
\text { administrativa }\end{array}$} & Aceptable & 37 & $25,2 \%$ \\
\hline & & Buena & 91 & $61,9 \%$ \\
\hline & & Excelente & 19 & $12,9 \%$ \\
\hline \multirow{14}{*}{ Dimensiones } & \multirow{3}{*}{ D1: Planeación } & Aceptable & 24 & $16,3 \%$ \\
\hline & & Buena & 82 & $55,8 \%$ \\
\hline & & Excelente & 41 & $27,9 \%$ \\
\hline & \multirow{4}{*}{ D2: Organización } & Deficiente & 7 & $4,8 \%$ \\
\hline & & Aceptable & 46 & $31,3 \%$ \\
\hline & & Buena & 55 & $37,4 \%$ \\
\hline & & Excelente & 39 & $26,5 \%$ \\
\hline & \multirow{4}{*}{ D3: Dirección } & Deficiente & 24 & $16,3 \%$ \\
\hline & & Aceptable & 45 & $30,6 \%$ \\
\hline & & Buena & 72 & $49,0 \%$ \\
\hline & & Excelente & 6 & $4,1 \%$ \\
\hline & \multirow{3}{*}{ D4: Control } & Deficiente & 20 & $13,6 \%$ \\
\hline & & Aceptable & 68 & $46,3 \%$ \\
\hline & & Bueno & 59 & $40,1 \%$ \\
\hline
\end{tabular}

Nota: Resultados obtenidos a partir de la aplicación de la encuesta y procesados con el programa estadístico SPSS.

En lo que respecta a la variable proceso presupuestal, los resultados a nivel descriptivo indican que un $51.0 \%$ considera que este es aceptable, un $31.3 \%$ considera que es bueno, un $12.9 \%$ considera que es excelente y un $4.8 \%$ considera que es deficiente, por tanto, en general la percepción de los trabajadores respecto al proceso presupuestal es considerada como de nivel medio.

En relación a las fases del proceso presupuestal, en lo que tiene que ver con programación el $29.9 \%$ considera que es buena, un $29.3 \%$ considera que aceptable, $22.4 \%$ considera que es deficiente y un $18.4 \%$ considera que es excelente, por lo que se puede afirmar que en general es considerado de nivel medio el proceso de programación del presupuesto en esta entidad pública; en la fase de formulación, los encuetados manifiestan un $37.4 \%$ que es buena, un $31.3 \%$ que es aceptable, un $26.5 \%$ considera que es excelente y un $4.8 \%$ considera que es deficiente, por lo que se puede considerar en general que la formulación del presupuesto es de nivel medio, pudiendo seguir mejorando; en lo que concierne a la aprobación del presupuesto, este se considera con calificativos de excelente por el 29,9\% de los encuestados, $29.9 \%$ considera a la vez que es aceptable, $24.5 \%$ considera que es bueno y $15.6 \%$ consideran que es deficiente, estos resultados indican que existen dos grupos uno de ellos que considera que la aprobación del presupuesto como adecuado o 
alto mientras que otro grupo considera que la aprobación del presupuesto tiene deficiencias.

Con respecto a la ejecución presupuestal, el $51 \%$ considera que es buena, un $23.8 \%$ considera que es aceptable, un $19.7 \%$ considera que es excelente y el $5.4 \%$ considera que es deficiente, por tanto se puede afirmar que se tiene mejores practicas en procesos de ejecución de presupuesto, debido a que se pone mayor atención o mejor interés; en lo que respecta a la evaluación del proceso presupuestal, el 34\% considera que es aceptable, $28.3 \%$ considera que es excelente, $27.2 \%$ considera que es buena, un $4.8 \%$ considera que es deficiente y un $4.8 \%$ considera que es muy deficiente, por lo que se puede afirmar que es mayormente de nivel medio la evaluación presupuestal. Los resultados se presentan en la table 2 .

\section{Tabla 2}

Resultados descriptivos de la variable proceso presupuestario y sus dimensiones

\begin{tabular}{|c|c|c|c|c|}
\hline & & Nivel & f & $\%$ \\
\hline \multirow{4}{*}{ Variable } & \multirow{4}{*}{$\begin{array}{l}\text { VD: Proceso } \\
\text { presupuestario }\end{array}$} & Deficiente & 7 & $4,8 \%$ \\
\hline & & Aceptable & 75 & $51,0 \%$ \\
\hline & & Bueno & 46 & $31,3 \%$ \\
\hline & & Excelente & 19 & $12,9 \%$ \\
\hline \multirow{21}{*}{ Dimensiones } & \multirow{4}{*}{ D1: Programación } & Deficiente & 33 & $22,4 \%$ \\
\hline & & Aceptable & 43 & $29,3 \%$ \\
\hline & & Buena & 44 & $29,9 \%$ \\
\hline & & Excelente & 27 & $18,4 \%$ \\
\hline & \multirow{4}{*}{ D2: Formulación } & Deficiente & 7 & $4,8 \%$ \\
\hline & & Aceptable & 46 & $31,3 \%$ \\
\hline & & Buena & 55 & $37,4 \%$ \\
\hline & & Excelente & 39 & $26,5 \%$ \\
\hline & \multirow{5}{*}{ D3: Aprobación } & Deficiente & 23 & $15,6 \%$ \\
\hline & & Aceptable & 44 & $29,9 \%$ \\
\hline & & Bueno & 36 & $24,5 \%$ \\
\hline & & Excelente & 44 & $29,9 \%$ \\
\hline & & Deficiente & 8 & $5,4 \%$ \\
\hline & \multirow{3}{*}{ D4: Ejecución } & Aceptable & 35 & $23,8 \%$ \\
\hline & & Buena & 75 & $51,0 \%$ \\
\hline & & Excelente & 29 & $19,7 \%$ \\
\hline & \multirow{5}{*}{ D5: Evaluación } & Muy deficiente & 7 & $4,8 \%$ \\
\hline & & Deficiente & 7 & $4,8 \%$ \\
\hline & & Aceptable & 50 & $34,0 \%$ \\
\hline & & Buena & 40 & $27,2 \%$ \\
\hline & & Excelente & 43 & $29,3 \%$ \\
\hline
\end{tabular}

Nota: resultados obtenidos a partir de la encuesta y procesados con el programa estadístico SPSS. 


\section{Influencia de la gestión administrativa en el proceso presupuestal}

El propósito principal de la investigación fue determinar la influencia de la gestión administrativa en el proceso presupuestal en el sector educación a nivel de gobierno subnacional, determinando a través de la regresión logística ordinal, con un grado de significancia del 0.00 menor a p=0.05, obteniendo un valor de Pseudo R-cuadrado, a través de la prueba de Nagelkerke de 0.918, que permite afirmar que la gestión administrativa explicaría el $91.8 \%$ del comportamiento de la variable proceso presupuestal. Los resultados se muestran en la tabla 3.

Tabla 3

Resultados de la influencia de la gestión administrativa en el proceso presupuestal

\begin{tabular}{lcccccc}
\hline Modelo & $\begin{array}{c}\text { Logaritmo de la } \\
\text { verosimilitud -2 }\end{array}$ & $\begin{array}{c}\text { Chi- } \\
\text { cuadrado }\end{array}$ & gl & Sig. & Pseudo R cuadrado \\
& & & & & $\begin{array}{c}\text { Cox y } \\
\text { Snell }\end{array}$ & 0,819 \\
& & & & & Nagelkerke & 0,918 \\
$\begin{array}{l}\text { Sólo } \\
\text { interceptación }\end{array}$ & 251,593 & & & & & \\
Final & 0,000 & 251,593 & 9 & 0,000 & McFadden & 0,767 \\
\hline
\end{tabular}

Función de enlace: Logit.

\section{Influencia de la gestión administrativa en la programación del proceso presupuestal}

A nivel especifico la investigación se propuso determinar la influencia de la gestión administrativa en la programación presupuestal, obteniendo como resultados del modelo un valor de chi cuadrado de 178.167, con un grado de significancia de 0.000 menor a $\mathrm{p}=0.05$, además de un Pseudo R cuadrado, calculado con la prueba de Nagelkerke de 0.751 , por lo que se puede afirmar que la gestión administrativa explica en $75.10 \%$ del resultado de la programación presupuestal. Como se puede evidenciar en la tabla 4.

\section{Tabla 4}

Resultados de la influencia de la gestión administrativa en la programación presupuestal

\begin{tabular}{|c|c|c|c|c|c|c|}
\hline Modelo & $\begin{array}{l}\text { Logaritmo de la } \\
\text { verosimilitud -2 }\end{array}$ & $\begin{array}{c}\text { Chi- } \\
\text { cuadrado }\end{array}$ & gl & Sig. & \multicolumn{2}{|c|}{ Pseudo R cuadrado } \\
\hline & & & & & $\begin{array}{l}\text { Cox y } \\
\text { Snell }\end{array}$ & 0,702 \\
\hline $\begin{array}{l}\text { Sólo } \\
\text { interceptación }\end{array}$ & 307,828 & & & & Nagelkerke & 0,751 \\
\hline Final & 129,660 & 178,167 & 9 & 0,000 & McFadden & 0,443 \\
\hline
\end{tabular}

Función de enlace: Logit. 


\section{Influencia de la gestión administrativa en la formulación del proceso presupuestal}

Del mismo modo se ha determinado la influencia de la gestión administrativa en la formulación del presupuesto, obteniendo como valor de Chi-cuadrado de 104,342 y un grado de significancia de 0.000, que es menor a p=0.05, además de un Pseudo R cuadrado, calculado a través de la prueba de Nagelkerke de 0.551, que permite afirmar que la gestión administrativa influye en un $55.10 \%$ del resultado de la formulación presupuestaria. Los resultados se muestran en la tabla 5 .

\section{Tabla 5}

Resultados de la influencia de la gestión administrativa en la formulación presupuestal

\begin{tabular}{lcccccc}
\hline Modelo & $\begin{array}{c}\text { Logaritmo de la } \\
\text { verosimilitud -2 }\end{array}$ & $\begin{array}{c}\text { Chi- } \\
\text { cuadrado }\end{array}$ & gl & Sig. & \multicolumn{2}{c}{ Pseudo R cuadrado } \\
& & & & & $\begin{array}{c}\text { Cox y } \\
\text { Snell }\end{array}$ & 0,508 \\
Sólo & 264,352 & & & & Nagelkerke & 0,551 \\
interceptación & 160,010 & 104,342 & 9 & 0,000 & McFadden & 0,277 \\
Final & Función de enlace: Logit. & & & &
\end{tabular}

\section{Influencia de la gestión administrativa en la aprobación del proceso presupuestal}

Por otro lado, también se ha medido la influencia de la gestión administrativa en la aprobación presupuestal, los resultados obtenidos indican un valor de Chi-cuadrado de 94,462 , con un grado de significancia de 0.000 , que es menor a $\mathrm{p}=0.05$, complementado con un Pseudo R cuadrado medido con la prueba de Nagelkerke de 0.508, que indica que la gestión administrativa explica el $50.8 \%$ del comportamiento de la aprobación presupuestal. Como se muestra en la tabla 6.

\section{Tabla 6}

Resultados de la influencia de la gestión administrativa en la aprobación presupuestal

\begin{tabular}{|c|c|c|c|c|c|c|}
\hline Modelo & $\begin{array}{l}\text { Logaritmo de la } \\
\text { verosimilitud -2 }\end{array}$ & $\begin{array}{c}\text { Chi- } \\
\text { cuadrado }\end{array}$ & gl & Sig. & \multicolumn{2}{|c|}{ Pseudo R cuadrado } \\
\hline & & & & & $\begin{array}{l}\text { Cox y } \\
\text { Snell }\end{array}$ & 0,474 \\
\hline $\begin{array}{l}\text { Sólo } \\
\text { interceptación }\end{array}$ & 318,404 & & & & Nagelkerke & 0,508 \\
\hline Final & 223,942 & 94,462 & 9 & 0,000 & McFadden & 0,237 \\
\hline
\end{tabular}

Función de enlace: Logit. 


\section{Influencia de la gestión administrativa en la ejecución del proceso presupuestal}

En relación a la influencia de la gestión administrativa en la ejecución presupuestal, se ha determinado un Chi-cuadrado de 69,480, un valor de significancia de 0.000 , que es menor a $\mathrm{p}=0.05$, además de un Pseudo R cuadrado obtenido a través de la prueba de Nagelkerke de valor 0.417 , que permite afirmar que la gestión administrativa explica el $41.7 \%$ del comportamiento de la ejecución presupuestal. Como se evidencia en la tabla 7.

\section{Tabla 7}

Resultados de la influencia de la gestión administrativa en la ejecución presupuestal

\begin{tabular}{lcccccc}
\hline Modelo & $\begin{array}{c}\text { Logaritmo de la } \\
\text { verosimilitud -2 }\end{array}$ & $\begin{array}{c}\text { Chi- } \\
\text { cuadrado }\end{array}$ & gl & Sig. & Pseudo R cuadrado \\
& & & & $\begin{array}{c}\text { Cox y } \\
\text { Snell }\end{array}$ & 0,377 \\
Sólo & 246,700 & & & Nagelkerke & 0,417 \\
interceptación & 177,221 & 69,480 & 9 & 0,000 & McFadden & 0,203 \\
Final & Función de enlace: Logit. & & &
\end{tabular}

\section{Influencia de la gestión administrativa en la evaluación del proceso presupuestal}

Finalmente, también fue el propósito determinar la influencia de la gestión administrativa en la evaluación presupuestal, obteniendo un valor de Chi-cuadrado de las variables de 100,556 , con un grado de significancia de 0.000 menor a $\mathrm{p}=0.05$, complementado con un Pseudo R cuadrado, determinado a través de la prueba de Nagelkerke de 0.530, por lo que se afirma que la gestión administrativa explica el 53\% de la variable evaluación presupuestal. Tal como se muestra en la tabla 8 .

\section{Tabla 8}

Resultados de la influencia de la gestión administrativa en la evaluación presupuestal

\begin{tabular}{lcccccc}
\hline Modelo & $\begin{array}{c}\text { Logaritmo de la } \\
\text { verosimilitud -2 }\end{array}$ & $\begin{array}{c}\text { Chi- } \\
\text { cuadrado }\end{array}$ & gl & Sig. & \multicolumn{2}{c}{ Pseudo R cuadrado } \\
\hline & & & & $\begin{array}{c}\text { Cox y } \\
\text { Snell }\end{array}$ & 0,495 \\
Sólo & 325,050 & & & Nagelkerke & 0,530 \\
interceptación & 224,493 & 100,556 & 9 &, 000 & McFadden & 0,250 \\
Final & Función de enlace: Logit. & & & &
\end{tabular}




\section{Discusión}

La gestión administrativa, sin duda es fundamental para realizar un adecuado proceso presupuestal en las instituciones del sector público. Es así que, si se desarrolla una efectiva gestión administrativa, por consiguiente, se debe lograr un excelente proceso presupuestal. En la nueva gestión pública se pone bastante énfasis en desarrollar una gestión con eficiencia, eficacia, economía, de calidad, con legitimidad y sostenibilidad (Funck \& Karlsson, 2020); para ello se debe contar con las personas adecuadas y la asignación de recursos suficientes que permitan lograr los objetivos y metas de la institución, con el afán de lograr cumplir su misión que le ha encargado la sociedad.

Del mismo modo lograr cumplir adecuadamente con cada uno de los pasos que concierne al proceso presupuestal en las entidades públicas, redundará en la prestación de mejores bienes, servicios e infraestructura para la sociedad en general; en el sector educación, específicamente en los gobiernos regionales, es indiscutible llevar a cabo este proceso de la mejor forma, ya que se trata de la atención de uno de los sectores más importantes, que en cuanto a servicios que brinda el estado se refiere. Es por eso que junto con el sector salud, son dos sectores en los que se viene trabajando con presupuestos orientados a resultados, es decir se cuenta con indicadores claros que permitan invertir adecuadamente los recursos asignados (Del_Pozo, 2017).

Los encuestados del sector educación en el gobierno regional estudiado, dan cuenta que la gestión administrativa es principalmente considerada como buena por un $61.9 \%$, esto pone de manifiesto el compromiso administrativo de los trabajadores con la institución, además que se sigue adecuadamente cada uno de los elementos. Es decir, se tiene una buena planificación, una adecuada organización de los recursos y actividades, una adecuada dirección de la institución y las áreas y un buen control que permite ir corrigiendo los errores y mejorando permanentemente. Resultados similares fueron los obtenidos por Cotrina (2017), quien encontró que la gestión administrativa es considerada como adecuada por un 51.4\%; Resultados aún más contundentes son los obtenidos por Riffo (2019), que obtuvo un nivel adecuado del 85\% en la gestión administrativa en el sector educación.

En lo que respecta al proceso presupuestal se considera aceptable por el $51 \%$ de los participantes del estudio, sustentado principalmente por el proceso de ejecución presupuestal como la dimensión más importante. Esto significa que existe adecuadas 
prácticas en el proceso, guiado principalmente por una nutrida normativa que van desde leyes, decretos, directivas y otro tipo de normas que apoyan en cada paso a seguir (DGPP, 2018). Los resultados son consistentes con los encontrados por Cotrina (2017), quien logró obtener un $47.6 \%$ de percepción como útil en la implementación del presupuesto en trabajadores del Ministerio de Educación.

Se ha determinado que en el sector educación de un gobierno subnacional, la gestión administrativa explica en un $91.8 \%$ el proceso presupuestal que allí se desarrolla. Es por ello que es fundamental seguir poniendo bastante énfasis en el proceso de gestión administrativa, con la finalidad de seguir mejorando cada paso del proceso presupuestal, debido a que si se tiene un mejor proceso presupuestal este redundará en una mejor educación de la sociedad en general, y en particular de los estudiantes en sus diversos niveles educativos. El resultado es similar al encontrado por Ayala (2018), quien encontró una relación del 0.729, entre estas mismas variables en un contexto de gobierno local. Del mismo modo es consistente con los resultados obtenidos por Del_Pozo (2017), quien encontró relación positiva entre la gestión administrativa y el presupuesto por resultados, quien concluye que es importante seguir impulsando la gestión administrativa con la finalidad de lograr mejores resultados en el sector público en general. Además es consistente con lo encontrado por Stack (2018), quien encontró que en contexto en desarrollo como es el caso peruano, la gestión administrativa es fundamental para tener éxito al momento de implementar políticas y evitando de esta manera fracasos de ellas, para ello entonces se debe procurar mejorar la autonomía y las diversas competencias administrativas de los servidores, del mismo modo se debe procurar la coordinación y la colaboración como mecanismos de mejora de la gestión administrativa, además de mecanismos adecuados de control que garanticen el uso adecuado de los recursos públicos.

Por tanto, se debe implementar políticas de reforzamiento permanente en capacidades de manejo administrativo en los servidores públicos, a fin de garantizar mejores procesos de gestión del presupuesto público, puesto que con ello se garantiza el cumplimiento de la misión de la institución en beneficio de la sociedad en su conjunto. Futuras investigaciones pueden incluir otros contextos a nivel del sector público, ya sea a nivel de ministerios o de gobiernos locales, así mismo se puede incluir diversas variables 
independientes para medir el efecto que tienen en la dinámica del proceso presupuestal en el Perú.

\section{CONCLUSIÓN O CONSIDERACIONES FINALES}

A modo de conclusión se puede precisar los siguiente:

La gestión administrativa es una variable muy importante para el proceso presupuestal, puesto que explica significativamente el comportamiento de esta variable, es por ello que los esfuerzos deben estar orientados a fortalecer las capacidades de los servidores públicos en temas de gestión administrativa a fin de que puedan garantizar el mejor uso de los fondos públicos.

La gestión administrativa, es básica para desarrollar cada una de las fases del proceso presupuestario, sin embargo se debe poner mayor énfasis en la fase de ejecución presupuestal, debido a que es la fase en la que se certifica el presupuesto, se compromete, devenga y gira los recursos públicos, es decir es la fase en la que realmente se da uso al presupuesto y se intercambia, ya sea con bienes, servicios, obras, o fuerza laboral a través del pago de remuneraciones.

En los gobiernos subnacionales al estar mayormente alejados de los ministerios se tiene deficiencias de acceso a la dinámica y cambiante normativa del sector público, es por ello que debe implementarse programas de actualización constante a fin de conocer y manejar de forma adecuada las modificaciones y como estas se deben aplicar en el proceso presupuestal.

\section{LISTA DE REFERENCIAS}

Abdulhakeem, A. (2020). Budget Transparency And Fiscal Performance: Lessons For Nigeria And Sri Lanka. i, 1-16.

Ayala, R. (2018). La gestión administrativa y su relación con el presupuesto en la municipalidad distrital de Los Olivos, 2018. In Tesis Para Obtener Titulo Profesional (Vol. 1). file:///C:/Users/MUNDO-PC/Downloads/marketing digital 2.pdf

Cotrina, L. R. (2017). La gestión administrativa en la implementación del presupuesto por resultados en el Ministerio de Educación. Lima 2017. In Universidad César Vallejo. https://hdl.handle.net/20.500.12692/9958

Del_Pozo, J. (2017). Gestión Administrativa y Presupuesto por Resultados en el Programa 0072 de la Comisión Nacional para el Desarrollo y Vida Sin Drogas, 
2017. In Sistema Nacional del Presupuesto.

https://www.mef.gob.pe/contenidos/presu_publ/capacita/guia_sistema_nacional_ presupuesto.pdf

DGPP. (2018). Informe de Seguimiento del Cumplimiento de Compromisos de las Evaluaciones de Diseño y Ejecución (EDEP) al 31 de diciembre de 2013. https://www.mef.gob.pe/contenidos/presu_publ/ppr/eval_indep/inf_seguimiento _compromisos2013.pdf

Esteban, N. (2021). Tipos de investigación. Http://Repositorio.Usdg.Edu.Pe/. http://repositorio.usdg.edu.pe/bitstream/USDG/34/1/Tipos-de-Investigacion.pdf

Funck, E. K., \& Karlsson, T. S. (2020). Twenty-five years of studying new public management in public administration: Accomplishments and limitations. Financial Accountability and Management, 36(4), 347-375. https://doi.org/10.1111/faam.12214

Hernández, R., Fernández, C., \& Baptista, P. (2014). Metodología de investigación (S. A. D. C. V. McGRAW-HILL / INTERAMERICANA EDITORES (ed.)). McGRAW-HILL / INTERAMERICANA EDITORES, S.A. DE C.V.

Hernández, R., \& Mendoza, C. (2018). Metodología de la investigación: las rutas cuantitativa, cualitativa y mixta. McGRAW-HILL / INTERAMERICANA EDITORES, S.A. DE C.

Hood, C. (1991). All Seasons? Public Administration, 69(1), 3-19. http://dx.doi.org/10.1111/j.1467-9299.1991.tb00779.x

Kesmodel, U. S. (2018). Cross-sectional studies - what are they good for? AOGS, 97, 388-393. https://doi.org/10.1111/aogs.13331

Makón, M. (2007). La Gestión por Resultados es sinónimo del presupuesto por resultados. XII Congreso Internacional Del CLAD, 23.

Massey, A. (2019). Persistent public management reform: an egregore of liberal authoritarianism? Public Money and Management, 39(1), 9-17. https://doi.org/10.1080/09540962.2018.1448160

Ministerio de Economía y Finanzas. (2021). Guia de orientación al ciudadano del $\begin{array}{ll}\text { presupuesto } & \text { públcio } 2021 .\end{array}$ https://www.mef.gob.pe/contenidos/presu_publ/documentac/guia_orientacion_ci udadano2021.pdf 
Navarro Manotas, E., Verbel Castellar, A., Robles García, D., \& Hurtado Ibarra, K. (2014). Regresión Logística Ordinal Aplicada a la Identificación de Factores de Riesgo para Cáncer de Cuello Uterino. Ingeniare, 17, 87. https://doi.org/10.18041/1909-2458/ingeniare.17.582

Pagani, M., \& Pantaleon, M. (2018). Presupuesto participativo : cambios en la gestión local y transformación de territorios en la ciudad de La Plata. Memoria Académica, 23.

Pineda, Ec., \& Pires, V. (2017). ¿A qué llaman hoy presupuesto participativo? GIGAPP Estudios Working Papers, 77, 435-449.

Riffo, R. (2019). Gestión administrativa y de calidad en los centros escolares de los Chorrillos.

$$
\text { Revista Scientific, }
$$

4 ,

$153-172$. https://doi.org/https://doi.org/10.29394/Scientific.issn.25422987.2019.4.E.9.153-172

Ríos, A. M., Benito, B., \& Bastida, F. (2017). Factors Explaining Public Participation in the Central Government Budget Process. Australian Journal of Public Administration, 76(1), 48-64. https://doi.org/10.1111/1467-8500.12197

Stack, K. (2018). The Office of Management and Budget: The Quarterback of EvidenceBased Policy in the Federal Government. Annals of the American Academy of Political and Social Science, 678(1), 112-123. https://doi.org/10.1177/0002716218768440

Taherdoost, H. (2020). Validity and Reliability of the Research Instrument; How to Test the Validation of a Questionnaire / Survey in a Research Hamed Taherdoost To cite this version : HAL Id : hal-02546799 Validity and Reliability of the Research Instrument; How to Test the .

Tuapanta, J., Duque, M., \& Mena, A. (2019). Alfa de Cronbach para validar un Cuestionario de uso de TIC TIC en Docentes Universitarios. Revista MktDescubre, 10(December 2017), 37-48.

Voloshyna, O. (2021). Modern methods of administrative management at the enterprises сучасні. International Periodic Scientific Journal, 7(2), 56-67. https://doi.org/10.30888/2663-5712 